\title{
Risk factors for under-five mortality in Ethiopia: Evidence from the 2016 Ethiopian Demographic and Health Survey
}

\author{
Y Berelie, ${ }^{1}$ MSc (Biostatistics); L Yismaw, ${ }^{2}$ MPH (Biostatistics); E Tesfa, ${ }^{1}$ MSc (Econometrics); M Alene, ${ }^{3}$ MPH (Biostatistics)
}

${ }^{1}$ Department of Statistics, Debre Markos University, Ethiopia

${ }^{2}$ Department of Public Health, Debre Markos University, Ethiopia

${ }^{3}$ Department of Statistics, Mizan-Tepi University, Tepi, Ethiopia

Corresponding author: Y Berelie (yebelay.ma@gmail.com)

\begin{abstract}
Background. The under-five child mortality (U5CM) rate is the most important sensitive indicator of the socioeconomic and health status of a community, and the overall development of a nation. Despite the world having made substantial progress in reducing child mortality since 1990, the global U5CM rate was 41 per 1000 in 2016. The rate is higher in Ethiopia than in several other low- and middle-income countries.

Objectives. To estimate the effects of socioeconomic and demographic factors on U5CM in Ethiopia.

Methods. A community-based cross-sectional study was conducted on 10641 under-five children. The 2016 Ethiopian Demographic and Health Survey data were used for this research. Binary logistic regression was employed to identify factors affecting the U5CM rate.

Results. The U5CM rate was 60 deaths per 1000 live births. Children who were delivered at home (adjusted odds ratio (aOR) 1.30; 95\% CI 1.04 - 1.63) and male (aOR 1.36; 95\% CI 1.15 - 1.60) were at an increased risk of death. Children whose family size was between 1 and 3 (aOR 5.54; 95\% CI 4.08 - 7.54), and 4 and 6 (aOR 1.94; 95\% CI 1.55 - 2.43) were more likely to die before age 5 than those whose family size was $\geq 6$. First-born (aOR 0.49; 95\% CI 0.36 - 0.67), second- or third-born (aOR 0.51; 95\% CI 0.39 - 0.67) and fourth- or fifth-born (aOR $0.71 ; 95 \%$ CI $0.56-0.91$ ) children were less likely to die than those who were sixth-born and above. Similarly, singleton children (aOR 0.20; 95\% CI 0.15 - 0.28), children residing in urban communities (aOR 0.55; 95\% CI 0.40 - 0.76) and children whose families had protected sources of water (aOR 0.84; 95\% CI 0.71 - 0.99) had reduced risks of death compared with their respective counterparts. Conclusions. The present study identified risk factors for under-five mortality in Ethiopia. Programmes to reduce under-five mortality in Ethiopia must focus on the place of delivery, households with unprotected sources of drinking water and families residing in rural areas.
\end{abstract}

S Afr J Child Health 2019;13(3):137-140. https://doi.org/10.7196/SAJCH.2019.v13i3.1645

Globally, an estimated 5.6 million children died before reaching their fifth birthday, with 15000 deaths occurring per day, in 2016. ${ }^{[1]}$ Despite substantial progress being made in reducing child mortality since 1990, the global nder-five child motality (U5CM) rate was estimated to be 41 per 1000 in 2016. ${ }^{[2]}$ Many developing countries still experience a high number of deaths among children below the age of $5 .{ }^{[3]}$ Consequently, children in these countries are 10 times more likely to die before the age of 5 years than children in developed countries. ${ }^{[4]}$

Over $80 \%$ of under-five deaths occur in low-income countries in sub-Saharan Africa and Southern Asia. It is estimated that over 60 million under-five children will die between 2017 and 2030, and more than half of these deaths will occur in sub-Saharan Africa and about 30\% in Southern Asia. In sub-Saharan Africa, 1 in 12 children dies before 6 months, which is 12 times higher than the average figure of 1 in 147 children in developed countries. ${ }^{[5]}$ The U5CM rate is estimated to be high (67 deaths per 1000 live births) in Ethiopia, which means that 1 in every 15 children dies before reaching the age of 5 years. ${ }^{[6]}$ This rate is higher than in several other low- and middleincome countries, and it is one of the challenges that the country needs to address.

Ethiopia aims to reduce the U5CM rate to 25 deaths per 1000 live births or below, and to end preventable deaths among children under age 5 by $20300^{[2,7]}$ Urgent efforts are needed to identify the risk factors, and to reduce under-five mortality. Understanding the causes of child mortality provides important public health insights. However, little is known about the magnitude and associated risk factors of child mortality in Ethiopia. Consequently, the present study helps to identify the strategies of child survival and to evaluate the country's progress towards the sustainable development goals. The study attempts to determine the magnitude and to identify the risk factors of U5CM in Ethiopia, using the 2016 EDHS data.

\section{Methods}

\section{Study design and setting}

A community-based cross-sectional study was conducted in regions of Ethiopia from January 18 to June 27 2016. The 2016 Ethiopian Demographic and Health Survey (EDHS) data were analysed in the present study. The survey was designed to provide estimates for the health and demographic variables of interest. The survey drew a representative sample of reproductive women aged $15-49$, by administering a questionnaire and making an anthropometric assessment of women and their children born within the previous 5 years. The EDHS employed a two-stage sample design to select respondents for the study. In the first stage, a total of 645 enumeration areas (EAs) (202 in urban areas and 443 in rural areas) were selected, with probability proportional to EA size. In the second stage, a fixed number of 28 households per cluster was selected, with an equal probability systematic selection from the newly created household list. A total of 18008 households were selected for the sample and 15683 eligible women were interviewed. Data on 10641 children aged under 5 years were generated from the interviewed women. Complete histories were collected on each child's birth and death. These data were used to identify the number of children born in the last 5 years and their respective ages at death. Retrospective information was obtained about children who had died in the last 
5 years based on information on all births to each woman within the 5 years preceding the survey.

\section{Variables and measurements}

The outcome variable was dichotomous ( $1=$ yes; $0=$ no). The independent variables were: child-related (age of child, sex of child, birth order of child, type of birth and place of delivery); parent-related (mother's place of residence - urban or rural); and household-related (family size or number of household members, household wealth index, source of drinking water and presence of toilet facility).

\section{Data analysis}

Multiple logistic regression analysis was employed to determine the factors independently influencing the U5CM rate. ${ }^{[8]}$ Maximum likelihood estimation was used to estimate the unknown model parameters.

Measures of goodness of fit are statistical tools used to explore the extent to which the fitted response compares with the observed data. Clearly, the fit is good if there is good agreement between the fitted and the observed data. Therefore, the model adequacy was assessed using the most commonly used methods of assessing likelihood, i.e. the ratio test and Hosmer-Lemeshow test. ${ }^{[8]}$
The analysis was performed using Statistical Analysis System software version 9.4 (SAS, USA). Variables with a $95 \%$ confidence interval (CI) for adjusted odds ratio (aOR) without including one were considered statistically significant predictors of U5CM. A letter of permission was obtained from the director of the Central Statistical Agency of Ethiopia, and data for this study were extracted from EDHS 2016.

\section{Results Descriptive results of predictor variables and under- five mortality}

Among the total of 10641 under-five children included in the study, the U5CM rate was 60 deaths per 1000 live births. The U5CM rate was distributed differently across different groups. Accordingly, the rates were 50 and 69 deaths per 1000 live births among male and female children, respectively. It was higher (209 deaths per 1000 live births) in children of a multiple birth than in singleton children (56 deaths per 1000 live births) (Table 1). Higher U5CM was observed in family sizes of between 1 and 3 (110 deaths per 1000 live births) and 4 and 6 (58 deaths per 1000 live births) compared with a family size $>6$ ( 46 deaths per 1000 live births). The U5CM rate varied with categories of family wealth index, falling from 69

Table 1. Descriptive results of predictor variables and U5CM from the 2016 EDHS ( $N=10641)$

\begin{tabular}{|c|c|c|c|c|c|}
\hline Variable & Total & No. of dead children & U5CM rate & $\chi^{2}(p$-value $)$ & $d f$ \\
\hline Gender & & & & & 1 \\
\hline Female & 5158 & 259 & 50 & $15.97(<0.000)$ & \\
\hline Male & 5483 & 376 & 69 & & \\
\hline Birth type & & & & & 1 \\
\hline Single birth & 10363 & 577 & 56 & $112.88(<0.000)$ & \\
\hline Multiple birth & 278 & 58 & 209 & & \\
\hline Birth order & & & & & 3 \\
\hline 1 st & 2167 & 136 & 63 & & \\
\hline $2 \mathrm{nd}$ or $3 \mathrm{rd}$ & 3338 & 176 & 53 & $5.76(0.124)$ & \\
\hline 4 th or 5 th & 2475 & 145 & 59 & & \\
\hline 6 th + & 2661 & 178 & 67 & & \\
\hline Family size & & & & & 2 \\
\hline $1-3$ & 1299 & 143 & 110 & & \\
\hline $4-6$ & 5286 & 304 & 58 & $72.10(<0.000)$ & \\
\hline$>6$ & 4056 & 188 & 46 & & \\
\hline Wealth index & & & & & 2 \\
\hline Poor & 5775 & 399 & 69 & $2132(50000)$ & \\
\hline Middle & 1466 & 80 & 55 & $21.33(<0.000)$ & \\
\hline Rich & 3400 & 156 & 46 & & \\
\hline Residence & & & & & 1 \\
\hline Urban & 1974 & 67 & 34 & $28.60(<0.000)$ & \\
\hline Rural & 8667 & 568 & 66 & & \\
\hline Place of delivery & & & & & 1 \\
\hline At home & 7271 & 490 & 68 & $24.36(<0.000)$ & \\
\hline At health facility & 3370 & 145 & 43 & & \\
\hline Source of water & & & & & 1 \\
\hline Protected & 6349 & 349 & 55 & $6.21(0.013)$ & \\
\hline Unprotected & 4292 & 286 & 67 & & \\
\hline Toilet facility & & & & & 1 \\
\hline Yes & 1790 & 85 & 48 & $5.70(0.017)$ & \\
\hline No & 8851 & 550 & 62 & & \\
\hline
\end{tabular}


to 46 deaths per 1000 live births in poor v. rich families, respectively. The U5CM rate was 66 and 34 deaths per 1000 live births in rural and urban communities, respectively. Similarly, the U5CM rate of children delivered at home and at a health facility was 68 and 43 deaths per 1000 live births, respectively. Moreover, the U5CM rate of children in households with unprotected water sources was higher $(67$ deaths per 1000 live births) than that of those who used water from a protected source (55 deaths per 1000$)$. The rate was also higher $(62$ deaths per 1000 live births) in families who did not have a standard toilet facility than those who had a standard toilet facility (48 deaths per 1000 live births). Based on $\chi^{2}$ test statistics, all independent variables considered in the study were significantly associated with a response variable at $25 \%$ level of significance (Table 1).

\section{Risk factors of U5CM}

A binary logistic regression model was used to analyse the determinants of U5CM. Details of the effect size of factors on odds of U5CM are depicted in Table 2.

Gender was a statistically significant predictor variable of U5CM. After adjusting other variables in the model, the odds of death were 1.36 times (aOR 1.36; 95\% CI 1.15 - 1.60) higher among male children than female children. Parity (birth order) of the last-born child was negatively associated with the odds of U5CM. The odds of U5CM among first-born children were 0.49 times (aOR 0.49; $95 \%$ CI 0.36 - 0.67), second- or third-born 0.51 times (aOR 0.51; $95 \%$ CI 0.39 - 0.67) and fourth- or fifth-born 0.71 times (aOR 0.71; 95\% CI 0.56 - 0.91) higher than those for sixth-born children and above. Lower odds of child mortality were found among children living in urban areas of Ethiopia. Children residing in urban areas were $45 \%$ less likely to die than those living in rural areas (aOR 0.55; $95 \%$ CI 0.40 - 0.76). Likewise, children who were delivered at home (aOR 1.30; 95\% CI 1.04 - 1.63) were 30\% more likely to die than those delivered at a health centre.

\section{Model adequacy checking}

The Hosmer-Lemeshow test was used to test model adequacy. The test statistic was 11.29 with a $p$-value of 0.19 , which showed that the model is a good fit to the data at $5 \%$ level of significance. The likelihood test statistic was also calculated to determine whether the fitted model was significant (the final model over the null model had a $p$-value $<0.0001$, which implied a good fit for the final model).

\section{Discussion}

The aim of the study was to identify the socioeconomic and demographic risk factors for U5CM in Ethiopia. The results showed that among 10641 under-five children considered, 635 (6\%) had died before celebrating their fifth birthday. The study revealed that sex of the child, age of the child, type of birth, birth order, family size, place of residence, place of delivery and source of drinking water were risk factors for U5CM in Ethiopia.

The U5CM rate was higher among male children. This result was in agreement with those of other studies. ${ }^{[9,10]}$ As there is no sex discrimination in healthcare and access to resources, the higher mortality rate among males is probably due to biological differences. Biologically, newborn boys are less mature than girls because circulating male hormones may contribute to their greater vulnerability to respiratory distress, intestinal infections and frequent congenital malformations. ${ }^{[11,12]}$

Singletons are less likely to die before age 5 than a child of a multiple birth. Owing to the fact that Ethiopia is a low-income country with limited resources, there may be poorer management of multiple births, who are often premature or display growth retardation.

The risk of death increases with an increase in birth order. This finding was consistent with other studies. ${ }^{[10,13-15]}$ An unexpected
Table 2. Risk factors of U5CM from the 2016 EDHS ( $N=10641)$

\begin{tabular}{|c|c|c|}
\hline Variables & $\mathrm{aOR}$ & 95\% CI for AOR \\
\hline Intercept & 0.12 & \\
\hline \multicolumn{3}{|l|}{ Gender } \\
\hline Male & 1.36 & $1.15-1.60$ \\
\hline \multicolumn{3}{|l|}{ Female (Ref.) } \\
\hline Age of child (months) & 1.02 & $1.01-1.02$ \\
\hline \multicolumn{3}{|l|}{ Birth type } \\
\hline Single & 0.20 & $0.15-0.28$ \\
\hline \multicolumn{3}{|l|}{ Multiple (Ref.) } \\
\hline \multicolumn{3}{|l|}{ Birth order } \\
\hline First & 0.49 & $0.36-0.67$ \\
\hline 2nd or 3rd & 0.51 & $0.39-0.67$ \\
\hline 4 th or 5 th & 0.71 & $0.56-0.91$ \\
\hline \multicolumn{3}{|l|}{$6 \mathrm{th}+($ Ref. $)$} \\
\hline \multicolumn{3}{|l|}{ Family size } \\
\hline $1-3$ & 5.54 & $4.08-7.54$ \\
\hline $4-6$ & 1.94 & $1.55-2.43$ \\
\hline \multicolumn{3}{|l|}{$>6$ (Ref.) } \\
\hline \multicolumn{3}{|l|}{ Wealth index } \\
\hline Poor & 1.10 & $0.88-1.39$ \\
\hline Middle income & 0.88 & $0.65-1.19$ \\
\hline \multicolumn{3}{|l|}{ Rich (Ref.) } \\
\hline \multicolumn{3}{|l|}{ Residence } \\
\hline Urban & 0.55 & $0.40-0.76$ \\
\hline \multicolumn{3}{|l|}{ Rural (Ref.) } \\
\hline \multicolumn{3}{|l|}{ Place of delivery } \\
\hline At home & 1.30 & $1.04-1.63$ \\
\hline \multicolumn{3}{|l|}{ At health facility (Ref.) } \\
\hline \multicolumn{3}{|l|}{ Water source } \\
\hline Protected & 0.84 & $0.71-0.99$ \\
\hline \multicolumn{3}{|l|}{ Unprotected (Ref.) } \\
\hline \multicolumn{3}{|l|}{ Toilet facility } \\
\hline Improved & 0.81 & $0.63-1.03$ \\
\hline \multicolumn{3}{|l|}{ Unimproved (Ref.) } \\
\hline $\begin{array}{l}\text { U5CM = under-five child mo } \\
\text { Health Survey; AOR = adjust } \\
\text { Ref. = reference category. }\end{array}$ & $\begin{array}{l}S=\text { Ethi } \\
; C I=c\end{array}$ & $\begin{array}{l}\text { emographic and } \\
\text { einterval; }\end{array}$ \\
\hline
\end{tabular}

finding was that the risk of U5CM decreased as family size of the household increased, meaning that children in a larger household had a better chance of surviving to see their fifth birthday. ${ }^{[9,12,16-18]}$ This is due to the fact that a mother who has had more than one child would have the experience to seek healthcare services to keep the child healthy.

U5CM was less likely to occur in children who live in urban than in rural areas. This result is in line with other studies. ${ }^{[3,13,17]}$ This might be due to the better quality of the health environment and sanitation in urban areas. In Ethiopia, political influence and inconsistent distribution of socioeconomic resources directly affects health conditions. The source of drinking water is also a risk factor for children's survival. Children who receive water from protected sources have a lower risk of death. This finding was in agreement with other studies. ${ }^{[14,19,20]}$

$\mathrm{U} 5 \mathrm{CM}$ is reduced if mothers deliver their children at a healthcare centre, where they receive appropriate assistance from skilled professionals during birth, as well as information on how to care for the child and the benefits of the healthcare system. ${ }^{[21]}$ 


\section{Conclusion}

The U5CM rate in Ethiopia was found to be associated with a number of factors, including multiple births, increasing birth order, place of residence, unprotected source of drinking water and place of delivery. The study suggests that the U5CM rate is still an problem in Ethiopia, and the findings will serve to inform policy makers. In the near future, programmes to reduce under-five mortality in Ethiopia must focus on home-based deliveries, households with unprotected sources of drinking water and residences in rural areas.

\section{Study limitations}

Since the source of data is secondary, not all factors expected to affect U5CM were available. The EDHS only collected data from women aged 15 - 49 who were alive in a given household. For mothers who had died, no data were collected. Similarly, no information was available regarding medical conditions.

Acknowledgements. We would like to acknowledge EDHS for allowing access to the 2016 EDHS dataset.

Author contributions. YB, LY, ET and MA participated in all steps of the study. YB has made substantive contributions to the acquisition, analysis, and interpretation of data. MA, LY and ET conceptualised the study, provided methodological guidance, and participated in drafting the manuscript. All authors read and approved the final manuscript.

\section{Funding. None.}

Conflicts of interest. None.

1. United Nations Children's Fund (UNICEF), World Health Organization, World Bank Group and United Nations. Levels and Trends in Child Mortality. Estimates Developed by the UN Inter-agency Group for Child Mortality Estimation (IGME). New York: UNICEF, 2017.

2. United Nations Children's Fund (UNICEF). Progress for Every Child in the SDG Era. New York: UNICEF, 2018.

3. Frissiano Ernest Honwana SFM. Socio-economic and demographic determinants of under-five mortality in Ethiopia. Open Public Health J 2018;11:1874-9445. https://doi.org/10.2174/1874944501710010160

4. Anteneh ZA, Andargie K, Tarekegn M. Prevalence and determinants of acute diarrhea among children younger than five years old in Jabithennan District, Northwest Ethiopia, 2014. BMC Pub Health 2017;17(1):99.
5. Adebowale SA, Morakinyo OM, Ana GR. Housing materials as predictors of under-five mortality in Nigeria: Evidence from 2013 Demographic and Health Survey. BMC Paediatr 2017;17(1):30. https://doi.org/10.1186/s12887-016-0742-3

6. Assefa Y DWV, Williams OD, Hill PS. Successes and challenges of the millennium development goals in Ethiopia: Lessons for the sustainable development goals. 2017:1-7.

7. Alene M. LY, Yebelay B., Bekalu K. Health care utilization for common childhood illnesses in rural parts of Ethiopia: Evidence from the 2016 Ethiopian Demographic and Health Survey. BMC Public Health 2019;19:57.

8. Hosmer DW Jr, Lemeshow S, Sturdivant RX. Applied Logistic Regression. New Jersey: John Wiley \& Sons, 2013.

9. Bedada D. Determinant of under-five child mortality in Ethiopia. Am J Stat Probabil 2017;6(4):198-204. https://doi.org/10.11648/j.ajtas.20170604.15

10. Ntenda PAM, Chuang K-Y, Tiruneh FN, Chuang Y-C. Factors associated with infant mortality in Malawi. J Experiment Clin Med 2014;6(4):125-131. https:// doi.org/10.1016/j.jecm.2014.06.005

11. Ezeh OK, Agho KE, Dibley MJ, Hall JJ, Page AN. Risk factors for postneonatal, infant, child and under- 5 mortality in Nigeria: A pooled cross-sectional analysis. BMJ Open 2015;5(3):e006779. https://doi.org/10.1136/bmjopen-2014-006779

12. Mulugeta FZ. Socioeconomic Factors of Early Childhood Mortality in Ethiopia: Evidence from Demographic and Health Survey. 2010. Addis Ababa: Ethiopian Economics Association, 2010.

13. Geneti KT, Deressa TK. Correlates of under-five child mortality in Ethiopia: A logistic regression analysis. Adv Appl Stat 2014;39(1):61.

14. Gebretsadik S, Gabreyohannes E. Determinants of under-five mortality in high mortality regions of Ethiopia: An analysis of the 2011 Ethiopia Demographic and Health Survey data. Int J Population Res 2016;2016:1602761. https://doi. org/10.1155/2016/1602761

15. Henry H, Hibbs J. Infant mortality and the size of the family. Pub Am Stat Assoc 1915;14(111):629-641. https://doi.org/10.2307/2964897

16. Bizuneh H, Getnet F, Meressa B, Tegene Y, Worku G. Factors associated with diarrheal morbidity among under-five children in Jigiga town, Somali Regional State, eastern Ethiopia: A cross-sectional study. BMC Pediatr 2017;17(1):182. https://doi.org/10.1186/s12887-017-0934-5

17. Kayode GA, Adekanmbi VT, Uthman OA. Risk factors and a predictive model for under-five mortality in Nigeria: Evidence from Nigeria Demographic and health survey. BMC Preg Childbirth 2012;12(1):10.

18. Mekonnen D. Infant and Child Mortality in Ethiopia: The role of socioeconomic demographic and biological factors in the previous five years period of 2000 and 2005. Lund: Lund University, 2011.

19. Wang L, Jacoby H. Environmental Determinants of Child Mortality in Rural China: A Competing Risks Approach. Washington: The World Bank, 2004.

20. Tariku DaD. Levels, trends and determinants of under-five mortality in Amhara region, Ethiopia using EDHS (2000 - 2011). J Health Med Nurs 2016;28.

21. Lugangira K, Kalokola F. Morbidity and mortality of children aged 2 - 59 months admitted in the Tanzania Lake Zone's public hospitals: A cross-sectional study. BMC Res Notes 2017;10(1):502. https://doi.org/10.1186/s13104-017-2818-Z

Accepted 11 April 2019. 